Revista de MatemáticA: TeOría y APliCACiones 2018 25(2) : 239-259

CIMPA - UCR ISSN: 1409-2433 (PRINT), 2215-3373 (ONLINE)

DOI: https://doi.org/10.15517/rmta.v25i2.33692

\title{
UNEXISTENCE OF LIMIT CYCLE IN AN OPTIMAL CONTROL PROBLEM OF A POPULATION \\ OF DIABETICS
}

\section{INEXISTENCIA DE CICLO LÍMITE EN UN \\ PROBLEMA DE CONTROL ÓPTIMO DE UNA POBLACIÓN CON DIABETES}

\author{
SÉVERINE BERNARD* TÉNISSIA CÉSAR ${ }^{\dagger}$ \\ SILVÈre P. NUIRO Allin PIÉTRUS $^{\S}$
}

Received: 21/Jun/2017; Revised: 7/May/2018;

Accepted: 16/May/2018

\begin{abstract}
Revista de Matemática: Teoría y Aplicaciones is licensed under a Creative Commons Reconocimiento-NoComercial-Compartirigual 4.0 International License. Creado a partir de la obra en $\mathrm{http}: / / \mathrm{www}$.revistas.ucr.ac.cr/index.php/matematica

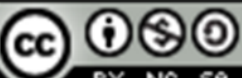

*Laboratoire de Mathématiques Informatique et Applications, Université des Antilles, Guadeloupe, France. E-Mail: severine.bernard@univ-antilles.fr

${ }^{\dagger}$ Misma dirección que/Same address as: S. Bernard. E-Mail: tenissia.cesar@univ-antilles.fr

${ }^{\ddagger}$ Misma dirección que/Same address as: S. Bernard. E-Mail: paul.nuiro@univ-antilles.fr

${ }^{\S}$ Misma dirección que/Same address as: S. Bernard. E-Mail: alain.pietrus@univ-antilles.fr
\end{abstract}




\begin{abstract}
This paper deals with one of the most important public health problem in the whole world that is diabetes, and more precisely its complications. From a model examining the complications or not of a population of diabetics, we associate a nonlinear optimal control problem. Considering the previous, we prove that the equilibrium state exists and is a saddle point. Moreover, we claim the unexistence of limit cycle in such a population, which is an interesting result concerning this world evil. Then we give some examples for which we characterize the equilibrium state which is not necessarily admissible.
\end{abstract}

Keywords: two-dimensional optimal control model; limit cycle; equilibrium state; Hopf bifurcation theorem.

\title{
Resumen
}

La diabetes, debido a sus complicaciones, es una de las enfermedades que más problemas plantean en la salud pública actual mundial. En este trabajo se parte de una población de diabéticos con y sin complicaciones y se asocia un problema de control óptimo no lineal que describe la dinámica de la población. Para este modelo se prueba la existencia del estado de equilibrio y que es un punto de ensilladura. Además se obtuvo que no existen ciclos límite, lo que es un resultado importante, dado el problema que se describe. Se presentan ejemplos para los cuales el estado de equilibrio que se caracteriza no es necesariamente admisible.

Palabras clave: modelo de control optimal bi-dimensional; ciclo límite; estado de equilibrio; teorema de bifurcación de Hopf.

Mathematics Subject Classification: 49J15, 34H05, 34H20, 90C46, 34C05.

\section{Introduction}

Diabetes is a chronic disease caused by a combination of hereditary and acquired bad factors, like family antecedents, overweight, unhealthy diet, physical inactivity. It occurs when the pancreas is not able to product insulin or when the body can not use effectively the insulin it makes. Consequently, the glucose from the diet stays in the blood instead of being transferred into the cells in order to produce energy. But a constant high glucose level in the blood causes a lot of damage to various organs and tissues, like kidney failure, blindness and eyes problems, heart attack, lower limb amputation and many other ones. The treatment is based on medication, healthy diet and physical exercises. According to the International Diabetes Federation, approximately 425 million adults 
were living with diabetes in the whole world in 2017, which caused 4 million deaths and 727 bilion USD in health expenditure. Moreover, 352 million people were at risk of developing diabetes. In particular, it concerned more than 3.5 million of persons in France in 2016 and more than 9 percent of the population of the archipelago of Guadeloupe, from the website of the French Federation of Diabetics. Its progression is stagerring in developed countries and even more in developing ones. Due to the mortality rate and the health expenditure caused by this evil, it is important to understand the triggering factors of the disease and its evolution.

There are in the literature many mathematical studies focused on this topic and everything related to it $[1,2,3,4,5,6,9,11,12]$. The different existing mathematical models are based on ordinary differential equations $[4,11,12]$, partial differential equations [11], delay differential equations [10, 11, 13, 14] and stochastic differential equations [11]. An overview of some mathematical models and software tools for the glucose-insulin regulatory system and diabetes has been done in [11]. In the last decades, some models focused on the intravenous glucose tolerance test, which consists to observe how a body reacts after the injection of a large amount of glucose [13], whereas other ones focused on the subcutaneous injection of insulin (see [17] and [9] for an overview). Another approach was to analyse the evolution of diabetes to the stage of complications [4] and an interesting one was given in [5] where the authors attempt to model mathematically the diabetes progression.

The blood glucose issue has also been seen as a control problem. In [6], J. R. Faria showed, by using the Hopf bifurcation theorem adapted to optimal control problem, that there is a cyclical behavior between the weight and the consumption of a diabetic created by the medical treatment. In [3], the authors considered an optimal control problem for the evolution of numbers of pre-diabetics and diabetics with and without complications and showed that the population of diabetics with complications decreases when an optimal control is applied. In [2], S. Bernard and A. Piétrus considered a new model of regulation adapted to the one introduced in [12] and studied it in the framework of ordinary differential equations and optimal control theory. By controlling the external glucose food intake, they proved that the plasma glycemia level can be minimized. In our previous work [1], we considered a population of diabetics, divided into two subcategories, one of diabetics with complications and another one of diabetics without complications as in [4]. From the model examining the complications of individuals diagnosed with diabetes, we associated a nonlinear optimal control problem, for which we proved that there is no cyclical behavior between the number of diabetics with complications, the one of diabetics without 
complications and the rate at which complications are healed. Moreover, as we considered a nonlinear optimal control problem with a scalar control and two states for which necessary conditions of an optimal control are well known, we could characterize the equilibrium point by using an adaptation of the Hopf bifurcation theorem to optimal control models as it has been done in $[7,18,19,20]$.

This work can be seen as a generalization of the previous one [1]. Indeed, we prove the existence of an optimal control and an equilibrium state whatever the controlled parameter and for any concave performance index, whereas in [1], it has been done in a particular case. Moreover, we are still able to claim that there is no limit cycle between the number of diabetics with complications and the one of diabetics without complications and that the equilibrium state is always a saddle point, which is an encouraging result since it reflects the unexistence of a back and forth between these two subcategories of diabetics, when the probability of developing complications or their cure rate are controlled.

Consequently, Section 2 is devoted to the study of the two dimensional nonlinear optimal control problem, that is we show the existence of an optimal control and characterize it via the Pontryagin's maximum principle. In Section 3, the stability analysis leads us to prove that the equilibrium state always exists and is a saddle point. In Section 4, by studying some suitable examples, we characterize the equilibrium state and see that it is not always admissible. We finish by some concluding remarks and perspectives.

\section{Optimal control problems in a population of diabetics}

We begin with the mathematical model of [4] in which we choose to put all the parameters depending on the time

$$
\left\{\begin{array}{l}
D^{\prime}(t)=I-(\lambda(t)+\mu(t)) D(t)+\gamma(t) C(t) \\
C^{\prime}(t)=\lambda(t) D(t)-(\gamma(t)+\mu(t)+\nu(t)+\delta(t)) C(t)
\end{array}\right.
$$

where:

- $t$ is the time,

- $D($.$) the number of diabetics without complications,$

- $C($.$) the number of diabetics with complications,$

- $I$ the incidence of diabetes,

- $\lambda($.$) the probability of developing a complication,$ 
- $\mu($.$) the natural mortality rate,$

- $\gamma($.$) the rate at which complications are healed,$

- $\nu($.$) the rate at which patients with complications become severely dis-$ abled,

- $\delta($.$) the mortality rate due to complications.$

Sometimes, we will omit the $t$ in order to relieve the writing. According to [4], this model has been constructed by considering a population in which $I$ cases are diagnosed with diabetes on a time interval. During this period of time, the number of diabetics without complications decreases in order to naturally death with rate $\mu$ or develop complications with rate $\lambda$, and also increases with the amount of diabetics with healed complications, with rate $\gamma$. In the same period of time, the number of diabetics with complications decreases with this last amount, the natural death, the mortality due to complications with rate $\delta$ and severely disabled patients with rate $\nu$ and increases by the coming of diabetics developing complications. The only one thing we add in this model is the fact that all the rates depend on the time, which seems more realistic.

Theoretically, any parameter of the model, that is $\lambda(),. \mu(),. \gamma(),. \nu($.$) or$ $\delta($.$) , can be chosen as control, but in practice, this is not possible since we can$ not control the natural mortality rate for example. In this state of minds, we can control the probability of developing complications by informing the diabetic patients on a necessary good lifestyle with physical activity and healthy diet. We can also control the rate at which complications are healed or the rate at which patients with complications become severely disabled, with an increase of health expenditure. Consequently, in the following, $u$ can be chosen as $\lambda($.$) ,$ $\gamma($.$) or \nu($.$) . The following result is a classical one and is just cited in order to$ well pose the optimal control problem.

Theorem 1 For all fixed control $u$, there exists one and only one maximal solution $\left(\left[0, t_{m}(u)\right], D_{u}(),. C_{u}().\right)$ of the Cauchy problem (1) with the initial conditions $D_{u}(0)=D_{0} \in \mathbb{R}_{+}, C_{u}(0)=C_{0} \in \mathbb{R}_{+}$and $t_{m}(u) \in \mathbb{R}_{+} \cup\{+\infty\}$.

Remark 1 The values of $D$ and $C$ will depend on the specific choice of the control $u$. We underline this dependence by using $D_{u}$ and $C_{u}$, instead of $D$ and $C$ respectively.

For a fixed discount rate $r>0$, our aim is to maximize the objective function

$$
\int_{0}^{+\infty} \exp (-r t) F(u(t), D(t), C(t)) d t
$$


where $F(u, D, C)$ is a concave performance index. In practice, our aim would be to minimize the complications in a population of diabetics. Consequently, $F$ can be a concave function, increasing with respect to $u=\gamma$ and/or $D$ and/or decreasing with respect to $u=\lambda, u=\nu$ and/or $C$. From these choices, we have this second classical result.

Theorem 2 Let $\left(D_{0}, C_{0}\right)$ be in $\mathbb{R}_{+}^{2}$ such that there is a control $u($.$) satisfying (1)$ with the initial conditions $D(0)=D_{0}$ and $C(0)=C_{0}$. There exists an optimal control $\bar{u}$ defined on $\left[0,+\infty\left[\right.\right.$ such that the associated trajectory $\left(D_{\bar{u}}(),. C_{\bar{u}}().\right)$ satisfies (1), the initial conditions and which maximizes

$$
\int_{0}^{+\infty} \exp (-r t) F(u(t), D(t), C(t)) d t
$$

In order to characterize this optimal control, we apply as usual the Pontryagin's maximum principle. We refer the reader to [15] and $[8,16,18]$ for more details and different applications of the optimal control theory.

Theorem 3 With previous assumptions, there exists an application $P()=.\left(P_{D}(),. P_{C}().\right):\left[0,+\infty\left[\rightarrow \mathbb{R}^{2}\right.\right.$ absolutely continuous called adjoint vector, such that, for almost all $t \geq 0$,

$$
\left\{\begin{array}{l}
P_{D}^{\prime}(t)=(r+\lambda(t)+\mu(t)) P_{D}(t)-\lambda(t) P_{C}(t)-\frac{\partial F}{\partial D}(u(t), D(t), C(t)), \\
P_{C}^{\prime}(t)=(r+\gamma(t)+\mu(t)+\nu(t)+\delta(t)) P_{C}(t)-\gamma(t) P_{D}(t)-\frac{\partial F}{\partial C}(u(t), D(t), C(t)),
\end{array}\right.
$$

with the limiting transversality conditions:

$$
\left\{\begin{array}{l}
\lim _{t \rightarrow+\infty} \exp (-r t) P_{D}(t) D(t)=0 \\
\lim _{t \rightarrow+\infty} \exp (-r t) P_{C}(t) C(t)=0
\end{array}\right.
$$


And the optimal control $\bar{u}$, whose existence has been proved in previous theorem, satisfies the following maximization's condition:

$$
H\left(\bar{u}, D, C, P_{D}, P_{C}\right)=\max _{v \in L^{\infty}\left(\mathbb{R}_{+}\right)} H\left(v, D, C, P_{D}, P_{C}\right)
$$

where $H$ is the associated Hamiltonian defined from the state equations and the concave performance index $F(u, D, C)$ as

$$
\begin{aligned}
H\left(u, D, C, P_{D}, P_{C}\right)= & F(u, D, C)+P_{D}[I-(\lambda+\mu) D+\gamma C] \\
& +P_{C}[\lambda D-(\gamma+\mu+\nu+\delta) C] .
\end{aligned}
$$

\section{Stability analysis}

This section is devoted to the existence proof of the equilibrium state and its classification. In our previous work [1], we fixed a control, a cost function and characterized the associated equilibrium state to see under which conditions it is admissible. In the present one, we prove that the equilibrium state exists whatever the control and the objective function.

Theorem 4 Whatever the control $u$ and whatever the concave performance index $F$ chosen as previously specified, there is an equilibrium state for the system

$$
\left\{\begin{array}{l}
D^{\prime}(t)=I-(\lambda(t)+\mu(t)) D(t)+\gamma(t) C(t) \\
C^{\prime}(t)=\lambda(t) D(t)-(\gamma(t)+\mu(t)+\nu(t)+\delta(t)) C(t) \\
P_{D}^{\prime}(t)=(r+\lambda(t)+\mu(t)) P_{D}(t)-\lambda(t) P_{C}(t)-\frac{\partial F}{\partial D}(u(t), D(t), C(t)) \\
P_{C}^{\prime}(t)=(r+\gamma(t)+\mu(t)+\nu(t)+\delta(t)) P_{C}(t)-\gamma(t) P_{D}(t)-\frac{\partial F}{\partial C}(u(t), D(t), C(t)) .
\end{array}\right.
$$

Proof. The equilibrium state $\left(D^{*}, C^{*}, P_{D}^{*}, P_{C}^{*}\right)$, if it exists, is solution of the following system

$$
\begin{cases}I-(\lambda(t)+\mu(t)) D(t)+\gamma(t) C(t) & =0 \\ \lambda(t) D(t)-(\gamma(t)+\mu(t)+\nu(t)+\delta(t)) C(t) & =0 \\ (r+\lambda(t)+\mu(t)) P_{D}(t)-\lambda(t) P_{C}(t)-\frac{\partial F}{\partial D}(u(t), D(t), C(t)) & 0 \\ (r+\gamma(t)+\mu(t)+\nu(t)+\delta(t)) P_{C}(t)-\gamma(t) P_{D}(t)-\frac{\partial F}{\partial C}(u(t), D(t), C(t)) & =0\end{cases}
$$


In order to have existence of an equilibrium state, we evaluate the determinant of this last system which is on the form

$$
S=\left|\begin{array}{cccc}
-(\lambda+\mu) & \gamma & 0 & 0 \\
\lambda & -(\gamma+\mu+\nu+\delta) & 0 & 0 \\
\cdot & \cdot & r+\lambda+\mu & -\lambda \\
\cdot & \cdot & -\gamma & r+\gamma+\mu+\nu+\delta
\end{array}\right|
$$

An easy computation gives

$$
\begin{aligned}
S= & {[\lambda(\mu+\nu+\delta)+\mu(\gamma+\mu+\nu+\delta)] } \\
& \cdot[(r+\mu)(r+\gamma+\mu+\nu+\delta)+\lambda(r+\mu+\nu+\delta)],
\end{aligned}
$$

which implies that $S \geq 0$ and $S$ is clearly equal to zero if and only if all the parameters of the model are equals to zero, since $S$ is a sum of non negative terms. Consequently, $S$ is strictly non negative, which implies the existence of an equilibrium state.

In order to characterize this equilibrium state, we have to choose the control and the objective function. We will see in the following section some examples for which we characterize it and note that it is not necessarily admissible.

Theorem 5 There is no limit cycle between the number of diabetics with complications, the one of diabetics without complications and the controlled parameter chosen as previously specified.

Proof. The steps leading to this result are similar to the ones of [1]. Let us define the Jacobian by

$$
J=\left(\begin{array}{cccc}
\partial D^{\prime} / \partial D & \partial D^{\prime} / \partial C & \partial D^{\prime} / \partial P_{D} & \partial D^{\prime} / \partial P_{C} \\
\partial C^{\prime} / \partial D & \partial C^{\prime} / \partial C & \partial C^{\prime} / \partial P_{D} & \partial C^{\prime} / \partial P_{C} \\
\partial P_{D}^{\prime} / \partial D & \partial P_{D}^{\prime} / \partial C & \partial P_{D}^{\prime} / \partial P_{D} & \partial P_{D}^{\prime} / \partial P_{C} \\
\partial P_{C}^{\prime} / \partial D & \partial P_{C}^{\prime} / \partial C & \partial P_{C}^{\prime} / \partial P_{D} & \partial P_{C}^{\prime} / \partial P_{C}
\end{array}\right)
$$

and a term $K$ by

$K=\left|\begin{array}{ll}\partial D^{\prime} / \partial D & \partial D^{\prime} / \partial P_{D} \\ \partial P_{D}^{\prime} / \partial D & \partial P_{D}^{\prime} / \partial P_{D}\end{array}\right|+\left|\begin{array}{cc}\partial C^{\prime} / \partial C & \partial C^{\prime} / \partial P_{C} \\ \partial P_{C}^{\prime} / \partial C & \partial P_{C}^{\prime} / \partial P_{C}\end{array}\right|+2\left|\begin{array}{cc}\partial D^{\prime} / \partial C & \partial D^{\prime} / \partial P_{C} \\ \partial P_{D}^{\prime} / \partial C & \partial P_{D}^{\prime} / \partial P_{C}\end{array}\right|$ 
In order to study the existence of a limit cycle, it is necessary to know the sign of the determinant of the Jacobian $J$ and of the term $K$ calculated at the equilibrium point. For our problem, we have

$$
J=\left(\begin{array}{cccc}
-(\lambda+\mu) & \gamma & 0 & 0 \\
\lambda & -(\gamma+\mu+\nu+\delta) & 0 & 0 \\
-\frac{\partial^{2} F}{\partial D^{2}} & -\frac{\partial^{2} F}{\partial C \partial D} & r+\lambda+\mu & -\lambda \\
-\frac{\partial^{2} F}{\partial D \partial C} & -\frac{\partial^{2} F}{\partial C^{2}} & -\gamma & r+\gamma+\mu+\nu+\delta
\end{array}\right)
$$

and

$$
\begin{aligned}
& K=\left|\begin{array}{cc}
-(\lambda+\mu) & 0 \\
-\frac{\partial^{2} F}{\partial D^{2}} & r+\lambda+\mu
\end{array}\right|+\left|\begin{array}{cc}
-(\gamma+\mu+\nu+\delta) & 0 \\
-\frac{\partial^{2} F}{\partial C^{2}} & r+\gamma+\mu+\nu+\delta
\end{array}\right| \\
&+2\left|\begin{array}{cc}
\gamma & 0 \\
-\frac{\partial^{2} F}{\partial C \partial D} & -\lambda
\end{array}\right|
\end{aligned}
$$

Even if the matrix $J$ and the term $K$ are more general than the ones of our previous study [1], we obtain exactly the same results for $\operatorname{det} J$ and $K$, that is

$$
\begin{aligned}
\operatorname{det} J= & {[\lambda(\mu+\nu+\delta)+\mu(\gamma+\mu+\nu+\delta)] } \\
& \cdot[(r+\mu)(r+\gamma+\mu+\nu+\delta)+\lambda(r+\mu+\nu+\delta)],
\end{aligned}
$$

and

$$
\begin{aligned}
K= & -(\lambda+\mu)(r+\lambda+\mu) \\
& -(\gamma+\mu+\nu+\delta)(r+\gamma+\mu+\nu+\delta)-2 \gamma \lambda .
\end{aligned}
$$

It is clear that $\operatorname{det} J \geq 0$ and $K \leq 0$, which leads to the result.

Theorem 6 The equilibrium state defined previously is a saddle point.

Proof. In order to classify the equilibrium state, we have to know the sign of $Q=\operatorname{det}(J)-\frac{1}{2} K^{2}$. Since $\operatorname{det}(J)$ and $K$ are exactly the same than those of [1], we used a similar method. 


\section{Characterization of the equilibrium state in particular cases}

In this section, we are going to characterize the equilibrium state, whose existence has been proved in the previous one, for different concave performance index. We will see that it is not always easy to characterize it and even if we can, it is not necessarily admissible taking into account the specificities of our problem. In the following, we consider the rate at which complications are healed as control, that is $u=\gamma$ and for a fixed discount rate $r>0$, the cost function we want to maximize is

$$
\int_{0}^{+\infty} \exp (-r t) F(u(t), D(t), C(t)) \mathrm{d} t
$$

where $F(u, D, C)$ is the concave performance index that we will fix at each case.

In the first example, we choose the control $u=\gamma$ and the performance index $F(u, D, C)=\ln u$. In this case, our goal is to control the rate at which complications are healed while maximizing it.

Proposition 1 If the control $u=\gamma$ and the performance index $F(u, D, C)=\ln u$ then the associated equilibrium state is not admissible.

Proof. According to Section 2, we have the existence of the optimal control leading to maximize the rate at which complications are cured. In order to characterize it, we use the Pontryagin's maximum principle which gives the existence of an adjoint vector $P()=.\left(P_{D}(),. P_{C}().\right):\left[0,+\infty\left[\rightarrow \mathbb{R}^{2}\right.\right.$ absolutely continuous such that, for almost all $t \geqslant 0$,

$$
\left\{\begin{array}{l}
P_{D}^{\prime}(t)=(r+\lambda(t)+\mu(t)) P_{D}(t)-\lambda(t) P_{C}(t), \\
P_{C}^{\prime}(t)=-\gamma(t) P_{D}(t)+(r+\gamma(t)+\mu(t)+\nu(t)+\delta(t)) P_{C}(t),
\end{array}\right.
$$

with the associated Hamiltonian

$$
H\left(\gamma, D, C, P_{D}, P_{C}\right)=\ln \gamma+P_{D}[I-(\lambda+\mu) D+\gamma C]+P_{C}[\lambda D-(\gamma+\mu+\nu+\delta) C],
$$

and the previously cited limiting transversality conditions. Consequently, the optimal control is

$$
\bar{\gamma}=\frac{1}{\left(P_{C}-P_{D}\right) C}
$$


Now, we try to characterize the associated equilibrium state of the system

$$
\left\{\begin{array}{l}
D^{\prime}(t)=I-(\lambda(t)+\mu(t)) D(t)+\gamma(t) C(t) \\
C^{\prime}(t)=\lambda(t) D(t)-(\gamma(t)+\mu(t)+\nu(t)+\delta(t)) C(t) \\
P_{D}^{\prime}(t)=(r+\lambda(t)+\mu(t)) P_{D}(t)-\lambda(t) P_{C}(t) \\
P_{C}^{\prime}(t)=-\gamma(t) P_{D}(t)+(r+\gamma(t)+\mu(t)+\nu(t)+\delta(t)) P_{C}(t),
\end{array}\right.
$$

by solving

$$
\begin{cases}I-(\lambda+\mu) D+\bar{\gamma} C & =0, \\ \lambda D-(\bar{\gamma}+\mu+\nu+\delta) C & =0, \\ (r+\lambda+\mu) P_{D}-\lambda P_{C} & =0 \\ -\bar{\gamma} P_{D}+(r+\bar{\gamma}+\mu+\nu+\delta) P_{C} & =0 .\end{cases}
$$

By replacing $\bar{\gamma}$ by $\frac{1}{\left(P_{C}-P_{D}\right) C}$, we have

$$
\begin{cases}I-(\lambda+\mu) D+\frac{1}{P_{C}-P_{D}} & =0 \\ \lambda D-(\mu+\nu+\delta) C-\frac{1}{P_{C}-P_{D}} & =0 \\ (r+\lambda+\mu) P_{D}-\lambda P_{C} & =0 \\ (r+\mu+\nu+\delta) P_{C}+\frac{1}{C} & =0\end{cases}
$$

The use of the first, fourth and second equations gives successively

$$
\begin{aligned}
D & =\frac{I\left(P_{C}-P_{D}\right)+1}{(\lambda+\mu)\left(P_{C}-P_{D}\right)}, \\
C & =-\frac{1}{(r+\mu+\nu+\delta) P_{C}},
\end{aligned}
$$

and

$$
\frac{\lambda I}{\lambda+\mu}+\frac{\mu+\nu+\delta}{(r+\mu+\nu+\delta) P_{C}}-\frac{\mu}{(\lambda+\mu)\left(P_{C}-P_{D}\right)}=0
$$


Moreover, with the third equation, we obtain

$$
P_{D}=\frac{\lambda}{r+\lambda+\mu} P_{C} \quad \text { so } \quad P_{C}-P_{D}=\frac{r+\mu}{r+\lambda+\mu} P_{C} .
$$

Consequently,

$$
D=\frac{I}{\lambda+\mu}+\frac{(r+\lambda+\mu)}{(r+\mu)(\lambda+\mu) P_{C}}
$$

and

$$
\frac{\lambda I}{\lambda+\mu}+\frac{\mu+\nu+\delta}{(r+\mu+\nu+\delta) P_{C}}-\frac{\mu(r+\lambda+\mu)}{(r+\mu)(\lambda+\mu) P_{C}}=0
$$

so

$$
P_{C}=\frac{r[\mu(r+\mu)-\lambda(\nu+\delta)]}{\lambda I(r+\mu)(r+\mu+\nu+\delta)} .
$$

Thus, the equilibrium state is

$$
\left\{\begin{array}{l}
D^{*}=\frac{I}{\lambda+\mu}+\frac{\lambda I(r+\lambda+\mu)(r+\mu+\nu+\delta)}{r(\lambda+\mu)[\mu(r+\mu)-\lambda(\nu+\delta)]}, \\
C^{*}=-\frac{\lambda I(r+\mu)}{r[\mu(r+\mu)-\lambda(\nu+\delta)]}, \\
P_{D}^{*}=\frac{r[\mu(r+\mu)-\lambda(\nu+\delta)]}{I(r+\mu)(r+\lambda+\mu)(r+\mu+\nu+\delta)}, \\
P_{C}^{*}=\frac{r[\mu(r+\mu)-\lambda(\nu+\delta)]}{\lambda I(r+\mu)(r+\mu+\nu+\delta)} .
\end{array}\right.
$$

Note that, if the term $\mu(r+\mu)-\lambda(\nu+\delta)$ is equal to zero then $P_{C}=P_{D}=0$ which is impossible since $\left(P_{D}, P_{C}\right)$ has to be non trivial. It follows that

$$
\gamma^{*}=-\frac{(r+\lambda+\mu)(r+\mu+\nu+\delta)}{r+\mu} .
$$

However, this equilibrium state is not admissible because $\gamma$ is a rate so has to be non negative.

In the second example, we choose the control $u=\gamma$ and the performance index $F(u, D, C)=\ln u+a \ln C$, with $a>0$. In this case, our goal is to control the rate at which complications are healed while maximizing the number of diabetics with cured complications. 
Proposition 2 If the control $u=\gamma$ and the performance index $F(u, D, C)=\ln u+a \ln C$, with $a>0$, then the associated equilibrium state is admissible if and only if $a>1$.

Proof. According to Section 2, we have the existence of the optimal control leading to maximize the number of diabetics with cured complications. In order to characterize it, we use the Pontryagin's maximum principle which gives the existence of an adjoint vector $P()=.\left(P_{D}(),. P_{C}().\right):\left[0,+\infty\left[\rightarrow \mathbb{R}^{2}\right.\right.$ absolutely continuous such that, for almost all $t \geqslant 0$,

$$
\left\{\begin{array}{l}
P_{D}^{\prime}(t)=(r+\lambda(t)+\mu(t)) P_{D}(t)-\lambda(t) P_{C}(t) \\
P_{C}^{\prime}(t)=-\gamma(t) P_{D}(t)+(r+\gamma(t)+\mu(t)+\nu(t)+\delta(t)) P_{C}(t)-\frac{a}{C(t)}
\end{array}\right.
$$

with the associated Hamiltonian

$$
\begin{aligned}
H\left(\gamma, D, C, P_{D}, P_{C}\right)= & \ln \gamma+a \ln C+P_{D}[I-(\lambda+\mu) D+\gamma C] \\
& +P_{C}[\lambda D-(\gamma+\mu+\nu+\delta) C]
\end{aligned}
$$

and the previously cited limiting transversality conditions. Consequently, the optimal control is

$$
\bar{\gamma}=\frac{1}{\left(P_{C}-P_{D}\right) C}
$$

We characterize the associated equilibrium state by solving

$$
\begin{cases}I-(\lambda+\mu) D+\frac{1}{P_{C}-P_{D}} & =0 \\ \lambda D-(\mu+\nu+\delta) C-\frac{1}{P_{C}-P_{D}} & =0 \\ (r+\lambda+\mu) P_{D}-\lambda P_{C} & =0 \\ (r+\mu+\nu+\delta) P_{C}+\frac{1-a}{C} & =0\end{cases}
$$

Rev.Mate.Teor.Aplic. (ISSN print: 1409-2433; online: 2215-3373) Vol. 25(2): 239-259, Jul-Dec 2018 
Similar computations give the following equilibrium state:

$$
\left\{\begin{array}{l}
D^{*}=\frac{I}{\lambda+\mu}\left[1+\frac{\lambda(r+\lambda+\mu)(r+\mu+\nu+\delta)}{(\mu+\nu+\delta)(a-1)(r+\mu)(\lambda+\mu)+\mu(r+\lambda+\mu)(r+\mu+\nu+\delta)}\right], \\
C^{*}=\frac{\lambda I(a-1)(r+\mu)}{(\mu+\nu+\delta)(a-1)(r+\mu)(\lambda+\mu)+\mu(r+\lambda+\mu)(r+\mu+\nu+\delta)}, \\
P_{D}^{*}=\frac{(\mu+\nu+\delta)(a-1)(r+\mu)(\lambda+\mu)+\mu(r+\lambda+\mu)(r+\mu+\nu+\delta)}{I(r+\mu)(r+\mu+\nu+\delta)(r+\lambda+\mu)} \\
P_{C}^{*}=\frac{(\mu+\nu+\delta)(a-1)(r+\mu)(\lambda+\mu)+\mu(r+\lambda+\mu)(r+\mu+\nu+\delta)}{\lambda I(r+\mu)(r+\mu+\nu+\delta)}
\end{array}\right.
$$

It follows that

$$
\gamma^{*}=\frac{(r+\lambda+\mu)(r+\mu+\nu+\delta)}{(r+\mu)(a-1)},
$$

and this equilibrium state is admissible if and only if $a>1$.

In the last example, we choose the control $u=\gamma$ and the performance index $F(u, D, C)=\alpha \ln u+a \ln C+K(D)$, with $a, \alpha>0$ and $K$ an increasing concave function of $D$. In this case, our goal is to control the rate at which complications are healed while maximizing the number of diabetics with cured complications and the number of diabetics without complications.

Proposition 3 Let us set $\sigma=\mu+\nu+\delta$ and assume that the control $u=\gamma$ and the performance index $F(u, D, C)=\alpha \ln u+a \ln C+K(D)$, with $a, \alpha>0$ and $K$ an increasing concave function of $D$. If $\mu=0$ and

- if $K(D)=D$ then, the associated equilibrium state is admissible if and only if $\alpha<a-\sigma^{-1} I$ and $r>\frac{\sigma I}{\sigma(a-\alpha)-I}$,

- or if $K(D)=\ln D$ and $a>\frac{\lambda}{\sigma}$ then, there is $\alpha^{*}$ satisfying

$$
0<\alpha^{*}<\min \left(a, \frac{a \sigma-\lambda}{2 \sigma+\lambda}\right)
$$

and $r^{*}>0$ such that the associated equilibrium state is admissible. 
Proof. As previously, we have the existence of the optimal control leading to maximize the number of diabetics with cured complications and the number of diabetic without complications. In order to characterize it, we use the Pontryagin's maximum principle which gives the existence of an adjoint vector $P()=.\left(P_{D}(),. P_{C}().\right):\left[0,+\infty\left[\rightarrow \mathbb{R}^{2}\right.\right.$ absolutely continuous such that, for almost all $t \geqslant 0$,

$$
\left\{\begin{array}{l}
P_{D}^{\prime}(t)=(r+\lambda(t)+\mu(t)) P_{D}(t)-\lambda(t) P_{C}(t)-K^{\prime}(D(t)) \\
P_{C}^{\prime}(t)=(r+\gamma(t)+\mu(t)+\nu(t)+\delta(t)) P_{C}(t)-\gamma(t) P_{D}(t)-\frac{a}{C(t)}
\end{array}\right.
$$

with the associated Hamiltonian

$$
\begin{aligned}
H\left(\gamma, D, C, P_{D}, P_{C}\right)= & \alpha \ln \gamma+a \ln C+K(D)+P_{D}[I-(\lambda+\mu) D+\gamma C] \\
& +P_{C}[\lambda D-(\gamma+\mu+\nu+\delta) C],
\end{aligned}
$$

and the previously cited limiting transversality conditions. Consequently, the optimal control is

$$
\bar{\gamma}=\frac{\alpha}{\left(P_{C}-P_{D}\right) C} \text {. }
$$

We characterize the equilibrium state by solving

$$
\begin{cases}I-(\lambda+\mu) D+\frac{\alpha}{P_{C}-P_{D}} & =0 \\ \lambda D-(\mu+\nu+\delta) C-\frac{\alpha}{P_{C}-P_{D}} & =0 \\ (r+\lambda+\mu) P_{D}-\lambda P_{C}-K^{\prime}(D) & =0 \\ (r+\mu+\nu+\delta) P_{C}+\frac{\alpha-a}{C} & =0\end{cases}
$$

In order to simplify the writing, let us set $\rho=\lambda+\mu$ and $T=P_{C}-P_{D}$. The use of the first and second equations gives

$$
D=\frac{I T+\alpha}{\rho T} \quad \text { and } \quad C=\frac{\lambda}{\sigma} D-\frac{\alpha}{\sigma T} .
$$

Consequently,

$$
C=\frac{\lambda(\alpha+I T)-\alpha \rho}{\sigma \rho T} .
$$

Moreover, by using the fourth equation, we obtain

$$
P_{C}=\frac{a-\alpha}{(r+\sigma) C} \quad \text { so } \quad P_{C}=\frac{(a-\alpha) \sigma \rho T}{(r+\sigma)[\lambda I T+\alpha(\lambda-\rho)]}
$$


Let us note that the quantity $\lambda I T+\alpha(\lambda-\rho)$ is equal to zero if and only if $C=0$ which has no sense in this situation. Moreover, the third equation implies that

$$
P_{D}=\frac{\lambda T \sigma \rho(a-\alpha)+(r+\sigma)[\lambda I T+\alpha(\lambda-\rho)] K^{\prime}(D)}{(r+\sigma)(r+\rho)[\lambda I T+\alpha(\lambda-\rho)]} .
$$

Thus

$T=\frac{(r+\rho)(a-\alpha) \sigma \rho T-\lambda T \sigma \rho(a-\alpha)-(r+\sigma)[\lambda I T+\alpha(\lambda-\rho)] K^{\prime}(D)}{(r+\sigma)(r+\rho)[\lambda I T+\alpha(\lambda-\rho)]}$

which is equivalent to

$$
\begin{aligned}
& \lambda I(r+\rho)(r+\sigma) T^{2} \\
& +\left[\alpha(\lambda-\rho)(r+\rho)(r+\sigma)-(r+\rho-\lambda)(a-\alpha) \sigma \rho+\lambda I K^{\prime}(D)(r+\sigma)\right] T \\
& +\alpha(r+\sigma)(\lambda-\rho) K^{\prime}(D)=0 .
\end{aligned}
$$

At this stage, it is not easy to characterize $T$ explicitly. We use the fact that $\mu=0$ that is $\lambda=\rho$ so

$$
T=\frac{\sigma(a-\alpha) r-I(r+\sigma) K^{\prime}(D)}{I(r+\lambda)(r+\sigma)}
$$

since $T$ is different from zero. Consequently, $T$ depends on the derivative of the function $K$. In order to finish the characterization, we consider two different cases. 
The first one is $K(D)=D$ for which we obtain

$$
T=\frac{\sigma(a-\alpha) r-I(r+\sigma)}{I(r+\lambda)(r+\sigma)}
$$

Consequently

$$
\left\{\begin{array}{l}
D^{*}=\frac{I}{\lambda}+\frac{\alpha I(r+\lambda)(r+\sigma)}{\lambda[\sigma(a-\alpha) r-I(r+\sigma)]} \\
C^{*}=\frac{I}{\sigma} \\
P_{D}^{*}=\frac{\lambda(a-\alpha) \sigma+(r+\sigma) I}{(r+\sigma)(r+\lambda) I} \\
P_{C}^{*}=\frac{(a-\alpha) \sigma}{(r+\sigma) I}
\end{array}\right.
$$

It follows that

$$
\gamma^{*}=\frac{\alpha \sigma(r+\lambda)(r+\sigma)}{\sigma(a-\alpha) r-I(r+\sigma)} .
$$

This equilibrium state is admissible if and only if $\sigma(a-\alpha) r-I(r+\sigma)>0$. As saying in [1], it occurs if and only if $\alpha<a-\sigma^{-1} I$ and $r>\frac{\sigma I}{\sigma(a-\alpha)-I}$.

The second case we consider is $K(D)=\ln D$ for which we obtain

$$
T=\frac{\sigma(a-\alpha) r D-I(r+\sigma)}{I(r+\lambda)(r+\sigma) D}
$$

Replacing $D$ by $\frac{I T+\alpha}{\rho T}$, we get

$$
(r+\lambda)(r+\sigma) I^{2} T^{2}+[(r+\sigma) \rho+\alpha(r+\lambda)(r+\sigma)-(a-\alpha) r \sigma] I T-(a-\alpha) \alpha r \sigma=0 .
$$

This is a polynomial equation on the form $p T^{2}+q T+l=0$ with

$$
\begin{aligned}
p & =(r+\lambda)(r+\sigma) I^{2}, \\
q & =[(r+\sigma) \rho+\alpha(r+\lambda)(r+\sigma)-(a-\alpha) r \sigma] I, \\
l & =r \alpha \sigma(\alpha-a) .
\end{aligned}
$$


If $a>\alpha$ then the associated discriminant $\Delta=q^{2}-4 l p$ is strictly non negative and consequently, there are two solutions

$$
T^{*}=\frac{1}{2 p}\left[-q \pm \sqrt{q^{2}-4 l p}\right] .
$$

If $q<0$ then there is one non negative solution $T^{*}$, which ensures the existence of an admisible equilibrium state. But $q$ is negative if and only if

$$
\alpha r^{2}+[\lambda+\alpha(\sigma+\lambda)+\sigma(\alpha-a)] r+\sigma \lambda(1+\alpha)<0
$$

wich occurs only when

$$
\lambda+\alpha(\sigma+\lambda)+\sigma(\alpha-a)<0,
$$

and for this, we need that $a \sigma>\lambda$ and $0<\alpha<\frac{a \sigma-\lambda}{2 \sigma+\lambda}$. Moreover, we remark that the discriminant of (2):

$$
\beta(\alpha)=[\lambda(1+\alpha)-a \sigma]^{2}-4 \alpha \sigma^{2}(a-\alpha),
$$

is such that

$$
\lim _{\alpha \rightarrow 0^{+}} \beta(\alpha)=[\lambda-a \sigma]^{2}>0
$$

So, there is $\alpha^{*}>0$ such that $\alpha^{*}<\min \left(a, \frac{a \sigma-\lambda}{2 \sigma+\lambda}\right)$ and $\beta\left(\alpha^{*}\right)>0$, since $\beta$ is a continuous function on $] 0, a\left[\right.$. Therefore, it exists $r^{*}>0$ solution of (2). Finally, the equilibrium state is

$$
\left\{\begin{array}{l}
D^{*}=\frac{1}{\lambda}\left[I+\frac{2 p \alpha}{-q+\sqrt{q^{2}-4 l p}}\right], \\
C^{*}=\frac{I}{\sigma}, \\
P_{D}^{*}=\frac{\lambda(a-\alpha) \sigma}{I(r+\sigma)(r+\lambda)}+\frac{\lambda\left(-q+\sqrt{q^{2}-4 l p}\right)}{(r+\lambda)\left[I\left(-q+\sqrt{q^{2}-4 l p}\right)+2 p \alpha\right]}, \\
P_{C}^{*}=\frac{(a-\alpha) \sigma}{I(r+\sigma)},
\end{array}\right.
$$

and it follows that

$$
\gamma^{*}=\frac{\alpha}{T^{*} C^{*}}=\frac{2 p \alpha \sigma}{I\left(-q+\sqrt{q^{2}-4 l p}\right)},
$$

which is admissible. 


\section{Concluding remarks}

This work is clearly a generalization of our previous one [1]. Considering a population of diabetics, we proved that there is no cyclical behavior between the number of diabetics with complications and the one of those without complications, when an optimal control is applied; that the equilibrium state always exists but is not always admissible and fortunately is always a saddle point. This result is an interesting one and reassures us since it reflects the fact that if we control the rate at which complications are healed, or the rate at which patients with complications become severely disabled or the probability of developing complications, by more health expenditure or a good lifestyle respectively, then there is no back and forth between the group of diabetics with complications and the one of diabetics without complications. In the following, it will be interesting to see how external actions like medication, food consumption or physical exercises can impact on the development of complications for diabetics. The first challenge would be to see how to include these parameters in the model and the second one to measure their impact on the disease and its evolution.

\section{Acknowledgments}

We would like to thank the anonymous referees for their valuable suggestions and remarks that enabled us to improve the presentation of this manuscript.

\section{References}

[1] Bernard, S.; Nuiro, S.P.; Piétrus, A. (2015) "Diabetes, complications and limit cycles", Applied Mathematics E-Notes 15: 197-206.

[2] Bernard, S.; Piétrus, A. (2015) "Optimal glucose modelling for diabetes", e-Journal of the Caribbean Academy of Sciences 8(1): 1-8.

[3] Boutayeb, A.; Boutayeb, W.; Lamlili, M. (2014) "Optimal control approach to the dynamics of population of diabetics", Applied Mathematical Sciences 8(56): 2773-2782.

[4] Boutayeb, A.; Chetouani, A.; Achouyab, A.; Twizell, E.H. (2006) "A nonlinear population model of diabetes mellitus", Journal of Applied Mathematics and Computing 21(1-2): 127-139.

[5] De Gaetano, A.; Hardy, T.; Beck, B.; Abu-Raddad, E.; Palumbo, P.; Bue-Valleskey, J.; Porksen, N. (2008) "Mathematical models of 
diabetes progression”, American Journal of Physiology, Endocrinology and Metabolism 295(6): E1462-E1479.

[6] Faria, J.R. (2003) "Limit cycles in an optimal control problem of diabetes", Applied Mathematics Letters 16(1): 127-130.

[7] Feichtinger, G.; Novak, A.; Wirl, F. (1994) "Limit cycles in intertemporal adjustment models: theory and applications", Journal of Economic Dynamics and Control 18(2): 353-380.

[8] Grass, D.; Caulkins, J.P.; Feichtinger, G.; Tragler, G.; Behrens, D.A. (2008) Optimal Control of Nonlinear Processes, With Applications in Drugs, Corruption, and Terror. Springer-Verlag Berlin Heidelberg, Germany.

[9] Li, J.; Johnson, J. D. (2009) "Mathematical models of subcutaneous injection of insulin analogues: a mini-review", Discrete and Continuous Dynamical Systems-Series B 12(2): 401-414.

[10] Li, J.; Kuang, Y. (2007) "Analysis of a model of the glucose-insulin regulatory system with two delays", SIAM Journal on Applied Mathematics 67(3): 757-776.

[11] Makroglou, A.; Li, J.; Kuang, Y. (2006) "Mathematical models and software tools for the glucose-insulin regulatory system and diabetes: an overview", Applied Numerical Mathematics 56(3-4): 559-573.

[12] Meilûnas, M. (1998) "On the blood glucose dynamics modelling", Mathematical Modelling and Analysis 3(1): 136-139.

[13] Mukhopadhyay, A.; De Gaetano, A.; Arino, O. (2004) "Modelling the intra-venous glucose tolerance test: a global study for a single-distributeddelay model", Discrete and Continuous Dynamical Systems. Series B 4(2): 407-417.

[14] Palumbo, P.; Pepe, P.; Panunzi, S.; De Gaetano, A. (2009) "Robust closedloop control of plasma glycemia: A discrete-delay model approach", Discrete and Continuous Dynamical Systems. Series B 12(2): 455-468.

[15] Pontryagin, L.; Boltyanski, V.; Gamkrelidze, R.; Michtchenko, E. (1974) Théorie mathématique des processus optimaux. Éditions Mir, Moscou.

[16] Trélat, E. (2008) Contrôle optimal: Théorie et applications. Vuibert, Paris, France. 
[17] Wang, H.; Li, J.; Kuang, Y. (2007) "Mathematical modelling and qualitative analysis of insuline therapies", Mathematical Biosciences 210(1): $17-33$.

[18] Wirl, F. (1992) "Cyclical strategies in two-dimensional optimal control models: necessary conditions and existence”, Annals of Operations Research 37(1): 345-356.

[19] Wirl, F. (1996) "Pathways to Hopf bifurcations in dynamic continuous-time optimization problems", Journal of Optimization Theory and Applications 91(2): 299-320.

[20] Wirl, F.; Feichtinger, G. (2010) "Modelling social dynamics (of obesity) and thresholds", Games 1(4): 395-414. 
